\title{
Book Rewiews Buchbesprechungen Livres nouveaux
}

G. Hofer und K.P. Kisker (Hrsg.): Die Sprache des Anderen. Gedenkschrift fúr Prof. Th. Spoerri. Bibliotheca psychiatrica, No. 154. Karger, Basel 1976. VIII + 189 pp., 19 fig., 1 tab.; SFr. 84.-. ISBN 38055-2346-7.

Der Band, der dem 1973 verstorbenen Prof. Dr. med. Dr. phil. Theodor Spoerri gewidmet ist, erinnert an Spoerris Arbeiten, wie „Genie und Krankheit” (1952), „Georg Trakl eine psychiatrisch anthropographische Untersuchung” (1954) und „Sprachphänomene und Psychose" (1964), um nur drei seiner Arbeiten zu nennen, die immer wieder die Aufmerksamkeit der Psychopathologen auf das Phänomen Sprache gerichtet haben. Gunter Hofer und Karl Peter Kisker haben den ausgezeichnet zusammengestellten Band herausge-geben und sind selbst mit Arbeiten über die Sprache der anderen vertreten.

\section{Zapotoczky, Wien}

Kommunkationsstörung ist vielleicht das zentralste Problem der Psychiatrie, da der Zugang zum Patienten sowohl in der Psychotherapie wie auch bei der Visite im ärztlichen Alltag ganz zentral auf die sprachliche Verständigung mit dem Kranken angewiesen ist. Verweigerung dieser Kommunikation im Mutismus oder Erschwerung durch Sprachentstel-lungen wie Formalismen, Deformationen, Symbolismen und Bedeutungsentleerungen, wie sie fur die Sprache Schizophrener charakteristisch sind, können daher zur Vereinsamung, ja zur völligen Isolation des Patienten fúhren, denn - und hier liegt der tragische Akzent - der Arzt kann dadurch verleitet werden, den krankheitsbedingten Persönlichkeitsabbau als viel schwerer einzustufen, als es der Realität entspricht, so dass der Arzt vor sich selbst die Entschuldigung hat, dass bei schweren Defektzuständen mit Medikament und Pflege-Versorgung das Auslangen gefunden werden könne - eine Selbsttäuschung, der er bei der Zahl der zu betreuenden Kranken aus Zeitersparnisgründen sehr leicht zum Opfer fällt.

Dass dies aber guten Gewissens nicht mehr möglich sein kann, beweisen die Arbeiten Navratils: In diesem Band wird am Beispiel eines seit 30 Jahren hospitalisierten Schizo-phrenen, des Patienten Alexander, der ausnahmslos nur auf Aufforderung und nach Themenstellung durch den Arzt ein umfangreiches literarisches Werk produziert hat, gezeigt, wie feinfúhlig und genau die Beobachtungen des Kranken den Spitalsalltag wiederzugeben imstande waren. Diesem Alexander sind auch die Beiträge von Roger Cardinal und Martin Almstedt gewidmet. Der Sprache der Schizophrenen gelten weiters die Beobachtungen von Mark und Inga Richartz, von Elka Spoerri, von W. Blankenburg und Günther Kapfhammer; N. Wolf hat Kurzgeschichten und Gedichte junger Drogenabhängiger vorgelegt, die ausnahmslos in der Entwöhnungsphase des Suchtprozesses entstanden sind; Manfred in der Beeck beschäftigt sich mit dem Rotwelsch, der Geheimsprache der Fahrenden, die sich durch eine fur den Aussenstehenden unverständliche Ausdrucksweise eine Sprachbarriere geschaffen haben, die ihnen Selbstbestätigung und Sicherheit zu bieten scheint.

Alfred und Françoise Brauner legen eine Studie über Sprachbruchstücke autistischer Kinder vor. Walter von Baeyer deckt die babylonische Sprachverwirrung als Ärgernis der Psychiatrie auf. Er 
weist darauf hin, dass die sprachliche Verständigung mit dem Patienten nicht dialogisch, sondern trilogisch ist, da je nach der Lehrmeinung der betreffenden Schule immer entweder Jaspers, Freud oder Binswanger usw. im Gespräch ,, mit dabei sind”. Jen-seits des Wortschatzes der jeweiligen Glossare aber verwendet er Schlüsselwörter (etwa: Begegnung - Identität Entfremdung usw.) als Brücke zwischen der „relativ exakten Wissenschaft” und der Umgangssprache des Patienten.

Book Reviews · Buchbesprechungen · Livres nouveaux

252

Mit Sprachinhaltsanalysen untersucht Gert Schäfer Psychotherapieverläufe, und W.T. Winkler beschreibt den Sprachzerfall bei einem Patienten, der wegen Depersonalisations-phänomenen in eine (innerhalb kurzer Zeit erfolgreiche) psychoanalytische Behand-lung kam.

Ein Eingehen auf alle Beiträge würde den Rahmen dieser Kurzbesprechung sprengen, daher möge der oben gegebene Überblick genügen. Seit den grundlegenden Arbeiten von Chomsky und von Lorenzer, von dem auch ein Beitrag über das Verhältnis von Sprache und Praxis in dem vorligenden Band enthalten ist, ist Sprachanalyse zum gemeinsamen Anliegen von Philologen, Psychiatern und Psychotherapeuten geworden.

Wenn Navratils Patient Theobald nach mehr als fünfzigjähriger Krankheit den Satz niedergeschrieben hat: „Normal reden ist eigentlich angenehm”, erfasst er damit ausge-zeichnet den Sachverhalt, dass es sowohl dem Kranken wohltut, verstanden zu werden, wie es auch für den Arzt die Mühe lohnt, sich durch alle psychotischen und neurotischen Sprach-barrieren nicht aufhalten zu lassen in dem leidenschaftlichen Bemühen, auch verstehen zu wollen und immer wieder urn sprachliche Verständigungsmöglichkeit zu ringen.

Ida Cermak, Wien

M. Mattila (ed): Alcohol, Drugs and Driving. Proceedings of the Satellite Symposium of the 6th International Congress of Pharmacopsychiatry on Alcohol, Drugs and Driving, Helsinki, July 1975. Modern Problems of Pharmacopsychiatry, vol. 11. Series Editors: Th. Ban, F.A. Freyhan, P. Pichot and W. Pöldinger. Karger, Basel 1976. XIII + 102 pp., 22 fig., 17 tab.; SFr. 49.-. ISBN 3-8055-2349-1.

Angesichts der Tatsache der hohen Rate von Verkehrsunfällen, insbesondere jedoch Verkehrstoten, in Zusammenhang mit akuter Alkoholisierung und der grossen Anzahl von Menschen, die Medikamente zu sich nehmen, welche in unterschiedlicher Weise die Verkehrstüchtigkeit beeinfl·ussen können, muss dieses Buch auf grösstes Interesse stossen. Bedingt durch verschiedene Beiträge, die sich jeweils mit einem Teilaspekt des Problems beschäftigen, ist dieser Sammelband zwar etwas uneinheitlich, zeigt jedoch gerade dadurch die Notwendigkeit intensivierter, international standardisierter Forschung auf: Die Angaben über tödliche Verkehrsunfálle, bei denen Alkoholisierung des Lenkers festgestellt werden konnte, sind in verschiedenen Ländern reichlich unpräzise und verbieten daher einen Vergleich. Noch schwieriger ist die Erfassung von Verkehrsunfällen unter Alkoholeinwir-kung, wenn durch den Unfall nur Sachschaden entstand. Angaben über die Häufigkeit von Alkoholisierung am Steuer fehlen völlig, vor allem, weil es zur Zeit kein praktikables Kontrollsystem gibt bzw. weil die gesetzlich möglichen Kontrollen im allgemeinen sehr wenig rigoros gehandhabt werden. In noch viel höherem Masse treffen diese Umstände für den Medikamentenmissbrauch zu, wobei in erster Linie, aber nicht ausschliesslich, den psychotropen Substanzen besonderes Augenmerk zukommt; es scheinen jedoch die Aus-wirkungen hinsichtlich der Unfallhäufigkeit weniger gravierend zu sein, wenn auch die Unfallhäufigkeit etwa doppelt so hoch ist wie bei Personen ohne Medikamenteneinwirkung. 
Eine finnische Studie konnte zeigen, dass zwar Medikamenteneinnahme kombiniert mit dem hohen Risikofaktor Alkohol eine zusätzliche Gefahr bedeutet, dass jedoch Personen, die unter der Wirkung von Medikamenten stehen, seltener in Unfälle verwickelt sind, aller-dings auch weniger fahren als jene, die häufig Alkohol trinken. Sehr interessant ist bei dieser Untersuchung das Ergebnis, dass psychiatrische Patienten, die nicht unter medikamentöser Therapie stehen, keine höhere Unfallsrate haben als entsprechende Kontrollgruppen.

Book Reviews · Buchbesprechungen · Livres nouveaux

253

Ein wichtiger Teü des Buches ist der Methodik zur Erfassung der Wirkung von Alkohol und Medikamenten auf gewisse psychomotorische Funktionen gewidmet, wobei auch die Relevanz psychometrischer Testverfahren für Fahrtauglichkeit bzw. Unfallhäufgkeit - unter anderem sehr verdienstvollerweise in einer Follow-Studie - untersucht wird. Eine Arbeits-gruppe aus den Vereinigten Staaten konnte zeigen, dass die Beeinträchtigung des Reaktions-vermögens durch Alkoholeinfluss unter Laborbedingungen geringer ausfällt als in vivo.

Den Abschluss des Buches bildet eine Liste von Medikamenten, die als Prämedikation, Kurzoder Lokalanästhetika Verwendung finden mit genauen Angaben, wie lange die Fahrtauglichkeit dadurch beeinträchtigt werden kann.

Wenn auch aufgrund der epidemiologischen Untersuchung im Interesse der Verkehrs-sicherheit der Ruf nach rigorosen Kontrollen und Massnahmen durchaus gerechtfertigt erscheint, so weisen doch die Autoren selbst auf die enormen Schwierigkeiten hin, die Effizienz verschiedener Massnahmen zu überprüfen und nachzuweisen.

G. Sonneck, Wien

D. Janz (ed.): Epileptology. Proceedings of the Seventh Symposium on Epilepsy, Berlin 1975.

Thieme, Stuttgart 1976. XX + 440 pp., 195 fig., 188 tab.; DM65.-. ISBN 3-13-5342-01-8.

Das 7. Internationale Epilepsiesymposium wurde 1975 in Westberlin abgehalten. Dieter Janz hat es übernommen, die auf diesem Symposium gehaltenen Vorträge einer Publikation zuzufúhren. Diese liegt nun unter dem angeführten Titel in englischer Sprache vor. Entspre-chend den Leitthemen des Symposiums gruppieren sich die Beiträge um zwei Schwer-punkte: zum einen um ,,Der Ambulante Epileptiker” mit den Untergruppen Prävention, F.pidemiologie, Naturgeschichte, Epilepsie und Psychose, Kind und Gesellschaft, Antiepilep-tika, Behandlung und Rehabilitation, zum anderen um „Methoden und Techniken der klinischen F, pileptologie” mit den Schwerpunkten Dokumentation klinischer Daten, kombi-nierte F.EG- und Videoaufnahme, diagnostische Techniken und Anwendung von EEG-Analysen in der Fpileptologie. In insgesamt 77 Beiträgen versuchen namhafte Epileptologen die erwähnten Themenkreise von den verschiedensten Standpunkten zu beleuchten. Aus der Vielfalt der so mitgeteilten Informationen wird die in der Schlussbemerkung von Janz angeführte Hoffnung verständlich, die F,pileptologie möge sich als eigenständiges Fach an den Universitätskliniken etablieren. Dennoch sollte dieses Buch nicht nur Epileptologen vorbehalten bleiben, zumal gerade die ambulante Betreuung von an Epilepsie leidenden Patienten niemals Aufgabe einiger weniger Spezialisten wird sein können.

P. Schuster, Wien

A. Langelüddeke und P.H. Bresser: Gerichtliche Psychiatrie; 4. Aufl. De Gruyter, Berlin 1976. XV+ 451 pp.; DM 98.-. ISBN 3-11-006777-3.

Das bekannte Lehrbuch von A. Langelüddeke wurde in einer neuen Auflage von P.H. Bresser bearbeitet, in vielen Kapiteln umgestellt und ergänzt. Die Ergänzungen betreffen vor allem Eignungsbegutachtung von Kraftfahrzeugen und ein Kapitel zur Psychologie des In-haftierten. 
Die Umarbeitung wurde auch durch eine geänderte Gesetzeslage notwendig. Das neue Massnahmenrecht und die teilweise Schuldausschliessung haben die Begutachtung schwierig gemacht, auch die Möglichkeit geboten, andere als den rein organischen Krank-

Book Reviews - Buchbesprechungen — Livres nouveaux

254

heitsbegriff von Schneider zur Anwendung zu bringen. Die Stellungnahme des Bearbeiters zu diesen Fragen ist eine konservative. Er meint, dass die Schlüssigkeit der Aussagen vormals eindeutig grosser war und dass ,theoretisch interessante Hypothesen und Konzepte voreilig als gesicherte Erkenntnis in die Begutachterpraxis übernommen worden sind". Die neue Gesetzeslage würde viel häufiger eine gutachterliche Stellungnahme, die ,ich weiss nicht” bedeuten würde, provozieren. Das Buch stellt im Zeitalter der Sammelbände, in denen Autoren nur begrenzte Wissensgebiete behandeln, als Werk eines einzigen Autors eine Rarität dar. Es ist dadurch vielleicht geschlossener als grosse Handbücher, kann aber notge-drungen gerade in den speziellen Kapiteln, wo es nicht um grundsätzliche Stellungnahmen geht, nicht den letzten Stand des Wissens vermitteln. So wird in dem Kapitel über die Psychologie des Inhaftierten eine ganz auf persönliche Empirie aufgebaute Psychopathologie vertreten (asozialer, antisozialer und pseudosozialer Verhaltensstil als Einteilungskriterien), von der aus etwa das ,funktionelle Psychosyndrom" als Haftreaktion in Frage gestellt wird. Soziologische Arbeiten über Subkulturen und gruppendynamische Mechanismen in geschlos-senen Institutionen (z.B. Goffman) werden nicht diskutiert.

Auch in dem Kapitel über Sexualdelinquenz überwiegen alte Literaturzitate, die Fülle neuer Ansätze in diagnostischer und therapeutischer Hinsicht (verhaltenstherapeutische, tiefenpsychologische bzw. biologische Aspekte) wird mit dem (richtigen) Hinweis auf ihren hypothetischen Charakter kaum gestreift.

Die grundlegend konservativen Stellungnahmen, die durch die Bearbeitungen Bressers noch deutlicher werden als bei Langelüddeke, müssen als engagierte Haltung gegen die Strömungen der Zeit gewertet werden.

Wolfgang Berner, Wien

K. Heinrich: Psychopharmaka in Klinik und Praxis. Thieme, Stuttgart 1976. VII + 123 pp., 17 fig., 9 tab.; DM 12.80. ISBN 3-13-5397-01-7.

In einer Zeit, in der die Anzahl der Psychopharmaka explosionsartig zunimmt, ist es nur zu begrüssen, wenn uns eine Reihe von Publikationen erreichen, die uns über diese so interessante Pharmakagruppe orientieren. Eine der jüngst erschienenen Veröffentlichungen auf diesem Gebiet ist Prof. Dr. Kurt Heinrichs ,, Psychopharmaka in •Klinik und Praxis”, das in Taschenbuchformat herausgegeben wurde. Das Büchlein gibt einen ausgezeichneten Über-blick über den praktischen Gebrauch der Psychopharmaka und informiert auch etwas über den heutigen Stand der Theorie der Pharmakopsychiatrie. Eingangs werden kurz die soma-tischpsychiatrischen Behandlungsmethoden vor Einführung der modernen Psychopharmaka, wie Dauerschlafbehandlung, Insulintherapie, chemische Heilkrampfbehandlung, elektrische Durchflutungsbehandlung, Stickstoffinhalation und Leukotomie erwähnt, und es wird auf deren Stellenwert in der modernen Psychiatrie hingewiesen. Sodann folgt das Hauptkapitel des Buches (,Psychopharmaka mit antipsychotischer Wirksamkeit”), wobei auf die ge-schichtliche Entwicklung, pharmakologische Daten und Hypothesen, biochemische Befunde, Einteilung und klinische Anwendung von Neuroleptika, Antidepressiva und Lithium einge-gangen wird.

Besonders wertvoll ist die detaillierte Behandlung der Indikationen, die auch dem Nichtpsychiater und dem Studenten eine rasche Information ermöglicht, welche psy- 
chopathologische Zustandsbilder er mit welchen Medikamenten in welcher Dosierung behandeln soil. Wertvoll erscheinen auch die Hinweise bezüglich der Dauer der Psychophar-

Book Reviews · Buchbesprechungen · Livres nouveaux

255

makabehandlung und des Einfl-usses der psychotropen Medikamente auf die Verkehrstauglichkeit sowie die Anführung der zu erwartenden Nebenerscheinungen und deren Gegenmassnahmen. Als nächstes Kapitel werden die Psychopharmaka mit anxiolytischer bzw. sedierender Wirksamkeit abgehandelt. Gemäss ihrem Stellenwert in der Psychopharmakotherapie werden Psychotonika und Psychodysleptika nur kurz gestreift. Nicht erwähnt werden hingegen Hypnotika, nootrope Substanzen und zentral durchblutungsfördernde Pharmaka, was angesichts der weiten Verbreitung dieser Medikamente gerade in der Praxis schade ist. Ein Präparateverzeichnis rundet das Buch ab, das alles in allem jedem Arzt und Studierenden eine Grundlage zur pharmakotherapeutischen Behandlung psychischer Erkrankungen in einer praxisbezogenen Form vermittelt. B. Saletu, Wien

American Psychiatric Association: Verhaltenstherapie in der Psychiatrie. UTB, vol. 614.

Steinkopff, Darmstadt 1976. XII + 116 pp., 1 fig.; DM 14.80. ISBN 3-7985-0453-9.

Verhaltenstherapie in der Psychiatrie - eine Broschüre der amerikanischen Fachgesell-schaft für Psychiatrie, in der die Ergebnisse einer Aufgabengruppe für Verhaltenstherapie festgehalten wurden; ein kurzer Leitfaden, der sich mit der Geschichte, den Definitionen, den grundlegenden Begriffen und Prinzipien, den Methoden der Verhaltenstherapie, den missverständlichen

Auffassungen über sie, ihrer Wirksamkeit und ihrer Beziehung zur dynamischen Psychiatrie, mit Problemen ihres Missbrauchs ebenso auseinandersetzt wie mit Fragen der Prävention psychischer Störungen oder der Notwendigkeit der Forschung. Die Aufgabengruppe, welche mit Verhaltenstherapeuten vom Range eines Ferster und Wolpe und Fachleuten wie Albee, Cameron, Kety in Kontakt gestanden ist, sollte die Aufmerk-samkeit amerikanischer Psychiater auf diese neue psychotherapeutische Richtuhg lenken; sie gelangte zur Ansicht, dass in der Anwendung der Verhaltenstherapie im Rahmen der psychiatrischen Klinik heute ein Standard erreicht wurde, der ,den informierten Praktikern im Dienste der mordernen klinischen und sozialen Psychiatrie fraglos viele Vorteile bieten" könne. 